Commun. Fac. Sci. Univ. Ank. Ser. A1 Math. Stat.

Volume 69, Number 1, Pages 183-192(2020)

DOI: $10.31801 /$ cfsuasmas.421435

ISSN 1303-5991 E-ISSN 2618-6470

http://communications.science.ankara.edu.tr/index.php?series=A1

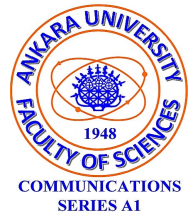

\title{
GENERALIZED PROJECTIVE CURVATURE TENSOR OF NEARLY COSYMPLECTIC MANIFOLD
}

\author{
NAWAF J. MOHAMMED AND HABEEB M. ABOOD
}

\begin{abstract}
In this paper, we concentrated our attention on geometry of generalized projective tensor of nearly cosymplectic manifold. In particular, we studied the flatness property of generalized projective tensor. This property helped us to find the necessary and sufficient condition that nearly cosymplectic manifold is a generalized Einstein manifold.
\end{abstract}

\section{INTRODUCTION}

One of the important curvature tensors is the projective tensor. According to this importance, many authors focused on its geometrical properties. Kirichenko 11 proved that nontrivial projective-recurrent $K$-space of maximal rank is 6dimensional manifold of constant curvature tensor. Abood [3] studied the projective tensor of nearly Kähler manifold. Abood and Mohammed [4 proved that almost Kähler manifold is a Kähler manifold if it is a projective parakähler manifold. Shashikala and Venkatesha 20, studied the generalized pseudo-projective $\Phi$-recurrent $N(k)$-contact metric manifold. Later on, Abood and Abd Ali [1] found the necessary condition that Viasman-Grey manifold has flat generalized projective tensor. Abood and Abd Ali 2] studied the projective-recurrent Viasman-Gray manifold. Finally, Atceken, Yildirim and Dirik [6], [7, 21, 22] studied certain curvature tensors including the pseudo-projective on some contact metric manifolds.

In this paper, we obtain some results on generalized projective tensor when it's act on nearly cosymplectic manifold. In particular, we found the necessary and sufficient conditions that nearly cosymplectic manifold is generalized Einstein manifold.

Received by the editors: June 20, 2018; Accepted: September 30, 2019

2010 Mathematics Subject Classification. 53C55, 53B35.

Key words and phrases. Nearly cosymplectic manifold, generalized projective curvature tensor, generalized Einstein manifold.

(C) 2020 Ankara University Communications Faculty of Sciences University of Ankara-Series A1 Mathematics and Statistics 


\section{Preliminaries}

Let $M$ be a smooth manifold of dimension $2 n+1$ greater than $3, X(M)$ be the module of smooth vector fields on $M, X^{c}(M)$ be the complexification of the module $X(M)$ and $T_{p}^{c}(M)$ be the complexification of tangent space $T_{p}(M)$ at the point $p \in M$.

An almost contact manifold ( $A C$-manifold) is the set $(M, \eta, \xi, \Phi, g)$, where $\eta$ is differential 1-form called a contact form, $\xi$ is a vector field called a characteristic, $\Phi$ is endomorphism of $X(M)$ called a structure endomorphisim and $g=\langle.,$.$\rangle is the$ Riemannian metric on $M$. Moreover, the following conditions are fulfilled:

$$
\eta(\xi)=1, \Phi(\xi)=0, \eta \circ \Phi=0, \Phi^{2}=-i d+\eta \otimes \xi
$$

and $\langle\Phi X, \Phi Y\rangle=\langle X, Y\rangle-\eta(X) \eta(Y) ; X, Y \in X(M)[8]$.

In the module $X^{c}(M)$, define two endomorphisms $\sigma$ and $\bar{\sigma}$ as follows: $\sigma=\frac{1}{2}(i d-\sqrt{-1} \Phi)$ and $\bar{\sigma}=-\frac{1}{2}(i d+\sqrt{-1} \Phi)$, then we can define two projections as follows:

$$
\Pi=\sigma \circ \ell=-\frac{1}{2}\left(\Phi^{2}-\sqrt{-1} \Phi\right) \text { and } \bar{\Pi}=\bar{\sigma} \circ \ell=\frac{1}{2}\left(\Phi^{2}+\sqrt{-1} \Phi\right),
$$

where $\sigma \circ \Phi=\Phi \circ \sigma=i \sigma$ and $\bar{\sigma} \circ \Phi=\Phi \circ \bar{\sigma}=-i \bar{\sigma}$. Therefore, If we denote $\operatorname{Im} \Pi=D_{\Phi}^{\sqrt{-1}}$ and $\operatorname{Im} \bar{\Pi}=D_{\Phi}^{-\sqrt{-1}}$, then

$$
X^{c}(M)=D_{\Phi}^{\sqrt{-1}} \oplus D_{\Phi}^{-\sqrt{-1}} \oplus D_{\Phi}^{0}
$$

where $D_{\Phi}^{\sqrt{-1}}, D_{\Phi}^{-\sqrt{-1}}$ and $D_{\Phi}^{0}$ are proper submodules of the endomorphism $\Phi$ with proper values $\sqrt{-1},-\sqrt{-1}$ and 0 respectively [13].

At each point $p \in M$, we can construct a frame in $T_{p}^{c}(M)$ by the form $\left(p, \varepsilon_{0}, \varepsilon_{1}, \ldots, \varepsilon_{n}\right.$, $\left.\varepsilon_{\hat{1}}, \ldots, \varepsilon_{\hat{n}}\right)$, where $\varepsilon_{a}=\sqrt{2} \sigma_{p}\left(e_{p}\right), \varepsilon_{\hat{a}}=\sqrt{2} \bar{\sigma}\left(e_{p}\right)$ and $\varepsilon_{0}=\xi_{p}$. The frame $\left(p, \varepsilon_{0}, \varepsilon_{1}, \ldots, \varepsilon_{n}\right.$, $\left.\varepsilon_{\hat{1}}, \ldots, \varepsilon_{\hat{n}}\right)$ is called $A$-frame [16].

The principle fiber of all $A$-frames with structure group $\{1\} \times U(n)$ is called an $G$-adjoined structure space.

The matrices of the $A C$-structure $\Phi_{p}$ and Riemannian metric $g_{p}$ in $A$-frame are given by the following forms:

$$
\left(\Phi_{j}^{i}\right)=\left(\begin{array}{ccc}
0 & 0 & 0 \\
0 & \sqrt{-1} I_{n} & o \\
0 & 0 & -\sqrt{-1} I_{n}
\end{array}\right),\left(g_{i j}\right)=\left(\begin{array}{ccc}
1 & 0 & 0 \\
0 & 0 & -I_{n} \\
0 & I_{n} & 0
\end{array}\right)
$$

where $I_{n}$ is the identity matrix of order $n[14$.

An almost contact manifold is called a nearly cosymplectic manifold ( $N C$-manifold) if the equality $\nabla_{X}(\Phi) Y+\nabla_{Y}(\Phi) X=0 ; X, Y \in X(M)$ holds 9 .

The following theorem explains the structure equations of $N C$-manifold in the $G$-adjoined structure space. 
Theorem 2.1. [15] In the G-adjoined structure space, the structure equations of $N C$-manifold are given by the following forms:

(1) $d \omega^{a}=\omega_{b}^{a} \wedge \omega^{b}+B^{a b c} \omega_{b} \wedge \omega_{c}+\frac{3}{2} C^{a b} \omega_{b} \wedge \omega ;$

(2) $d \omega_{a}=-\omega_{a}^{b} \wedge \omega_{b}+B_{a b c} \omega^{b} \wedge \omega^{c}+\frac{3}{2} C_{a b} \omega^{b} \wedge \omega ;$

(3) $d \omega=C^{b c} \omega_{b} \wedge \omega_{c}+C_{b c} \omega^{b} \wedge \omega^{c}$;

(4) $d \omega_{b}^{a}=\omega_{c}^{a} \wedge \omega_{b}^{c}+\left[A_{b c}^{a d}-2 B^{a d h} B_{h b c}+\frac{3}{2} C^{a d} C_{b c}\right] \omega^{c} \wedge \omega_{d}$,

where $B^{a b c}=\frac{\sqrt{-1}}{2} \Phi_{\hat{b}, \hat{c}}^{a}, C^{a b}=\sqrt{-1} \Phi_{0, \hat{b}}^{a}, C_{a b}=-\sqrt{-1} \Phi_{b, 0}^{\hat{a}}$ and $B_{a b c}=-\frac{\sqrt{-1}}{2} \Phi_{b, c}^{\hat{a}}$. The tensors $B, C$ and $A$ are called the first, second and third structure tensors respectively.

Definition 2.1. 17] A Riemann-Christoffel tensor $R$ of a smooth manifold $M$ is a tensor of type $(4,0)$ which is defined by

$$
R(X, Y, Z, W)=g(R(Z, W) Y, X),
$$

where $R(X, Y) Z=\left(\left[\nabla_{X}, \nabla_{Y}\right]-\nabla_{[X, Y]}\right) Z$, and has the following properties:

(1) $R(X, Y, Z, W)=-R(Y, X, Z, W)$;

(2) $R(X, Y, Z, W)=-R(X, Y, W, Z)$;

(3) $R(X, Y, Z, W)=R(Z, W, X, Y)$;

(4) $R(X, Y, Z, W)+R(X, Z, W, Y)+R(X, W, Y, Z)=0$.

The components of Riemann-Christoffel tensor of $N C$-manifold are given in theorem below.

Lemma 2.1. [15] In the G-adjoined structure space, the components of RiemannChristoffel tensor of NC-manifold have the following forms:

(1) $R_{\hat{a} b c d}=0$;

(2) $R_{a b c d}=-2 B_{a b[c d]}$;

(3) $R_{\hat{a} \hat{b} c d}=-2 B^{a b h} B_{h c d}$;

(4) $R_{\hat{a} 0 b 0}=C^{a c} C_{b c}$;

(5) $R_{\hat{a} b c \hat{d}}=A_{b c}^{a d}-B^{a d h} B_{h b c}-\frac{5}{3} C^{a d} C_{b c}$.

The other components of Riemann-Christoffel tensor $R$ can be obtained by the property of symmetry for $R$ or equal to zero.

Definition 2.2. 10 A generalized Riemannian curvature tensor $G_{R}$ on $N C$-manifold $M$ is a tensor of type $(4,0)$ which is defined as the following form: $G_{R}(X, Y, Z, W)=\frac{1}{16}\{3[R(X, Y, Z, W)+R(\Phi X, \Phi Y, Z, W)+R(X, Y, \Phi Z, \Phi W)+$ $R(\Phi X, \Phi Y, \Phi Z, \Phi W)]-R(X, Z, \Phi W, \Phi Y)-R(\Phi X, \Phi Z, W, Y)-R(X, W, \Phi Y, \Phi Z)-$ $R(\Phi X, \Phi W, Y, Z)+R(\Phi X, Z, \Phi W, Y)+R(X, \Phi Z, W, \Phi Y)+R(\Phi X, W, Y, \Phi Z)+R(X, \Phi W, \Phi Y, Z)\}$, where $R(X, Y, Z)$ is the Riemann-Christoffel tensor, $X, Y, Z, W \in T_{p}(M)$ and has the following properties:

(1) $G_{R}(X, Y, Z, W)=-G_{R}(Y, X, Z, W)=-G_{R}(X, Y, W, Z)$;

(2) $G_{R}(X, Y, Z, W)=G_{R}(Z, W, X, Y)$;

(3) $G_{R}(X, Y, Z, W)+G_{R}(X, Z, W, Y)+G_{R}(X, W, Y, Z)=0$; 
(4) $G_{R}(X, \Phi X, \Phi X, X)=R(X, \Phi X, \Phi X, X)$.

Definition 2.3. 18] A tensor $G_{r}$ of type $(2,0)$ which is defined as $\left(G_{r}\right)_{i j}=\left(G_{R}\right)_{i j k}^{k}$ is called a generalized Ricci tensor.

Remark 2.1. [18] A generalized Ricci tensor is symmetric, this follows form the properties of symmetry of generalized Riemannian curvature tensor. This mean $\left(G_{r}\right)_{i j}=\left(G_{r}\right)_{j i}$.

Definition 2.4. A generalized projective tensor $G_{P}$ is a tensor of type $(4,0)$ which is defined as the form:

$$
\left(G_{P}\right)_{i j k l}=\left(G_{R}\right)_{i j k l}-\frac{1}{2 n}\left[\left(G_{r}\right)_{i k} g_{j l}-\left(G_{r}\right)_{j k} g_{i l}\right] .
$$

Definition 2.5. [11] Let $M$ be an AC-manifold, an $\Phi$-holomorphic sectional curvature ( $\Phi H S$-curvature) of a manifold $M$ in the direction $X \in X(M), X \neq 0$ is a function $H(X)$ which is defined as:

$$
H(X)=\langle R(X, \Phi X) X, \Phi X\rangle\|X\|^{-4}
$$

Definition 2.6. 11] An AC-manifold is called a manifold of point constant $\Phi H S$ curvature if

$$
\langle R(X, \Phi X) X, \Phi X\rangle=c\|X\|^{4},
$$

where $c \in C^{\infty}(M)$, for all $X \in X(M)$

Theorem 2.2. 11] An AC-manifold is a manifold of point constant $\Phi H S$-curvature $C_{0}$ if and only if, on the $G$-adjoined structure, the following equation holds:

$$
R_{(b c)}^{(a d)}=\frac{C_{0}}{2} \tilde{\delta}_{b c}^{a d},
$$

where $C_{0} \in C^{\infty}(M)$ and $\tilde{\delta}_{b c}^{a d}=\delta_{b}^{a} \delta_{c}^{d}+\delta_{c}^{a} \delta_{b}^{d}$.

Definition 2.7. 19] A Riemannian manifold is called an Einstein manifold, if the Ricci tensor satisfies the equation $r_{i j}=e g_{i j}$, where e is an cosmological constant.

Similar to the above definition, we can introduce the following definition.

Definition 2.8. A Riemannian manifold is called a generalized Einstein manifold, if the generalized Ricci tensor satisfies the equation $\left(G_{r}\right)_{i j}=\left(G_{e}\right) g_{i j}$, where $G_{e}$ is a generalized cosmological constant.

\section{The main Results}

In this section, we calculated the components of the generalized Riemannian curvature tensor. Moreover, the necessary and sufficient condition that a nearly cosymplectic manifold is generalized Einstein manifold has been found.

Lemma 3.1. In the G-adjoined structure space, the components of the generalized

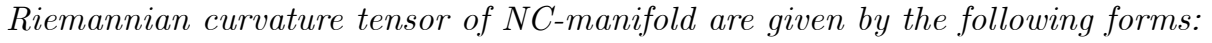


(1) $\left(G_{R}\right)_{\hat{a} b \hat{c} d}=-A_{b d}^{a c}$;

(2) $\left(G_{R}\right)_{\hat{a} b c \hat{d}}=-\frac{1}{2}\left[A_{b c}^{a d}-3 B^{a d h} B_{h b c}-\frac{5}{3} C^{a d} C_{b c}\right]$.

And the others are conjugate to the above components or equal to zero.

Proof: By using the Lemma 2.1 and Definition 2.2, we compute the components of generalized projective tensor as the following:

1) Put $i=\hat{a}, j=b, k=\hat{c}$ and $l=d$, we have

$$
\begin{gathered}
\left(G_{R}\right)_{\hat{a} b \hat{c} d}=\frac{1}{16}\left\{3\left[R_{\hat{a} b \hat{c} d}+R_{\hat{a} b \hat{c} d}+R_{\hat{a} b \hat{c} d}+R_{\hat{a} b \hat{c} d}\right]+R_{\hat{a} \hat{c} d b}+R_{\hat{a} \hat{c} d b}-R_{\hat{a} d b \hat{c}}-\right. \\
\left.R_{\hat{a} d b \hat{c}}+R_{\hat{a} \hat{c} d b}+R_{\hat{a} \hat{c} d b}-R_{\hat{a} d b \hat{c}}-R_{\hat{a} d b \hat{c}}\right\}
\end{gathered}
$$

Making use of the properties of $G_{R}$, we get

$$
\left(G_{R}\right)_{\hat{a} b \hat{c} d}=-A_{b d}^{a c} \text {. }
$$

2) Put $i=\hat{a}, j=b, k=\hat{c}$ and $l=d$, we obtain

$$
\begin{gathered}
\left(G_{R}\right)_{\hat{a} b \hat{c} d}=\frac{1}{16}\left\{3\left[R_{\hat{a} b \hat{c} d}+R_{\hat{a} b \hat{c} d}+R_{\hat{a} b \hat{c} d}+R_{\hat{a} b \hat{c} d}\right]+R_{\hat{a} \hat{c} d b}+R_{\hat{a} \hat{c} d b}-R_{\hat{a} d b \hat{c}}-\right. \\
\left.R_{\hat{a} d b \hat{c}}+R_{\hat{a} \hat{c} d b}+R_{\hat{a} \hat{c} d b}-R_{\hat{a} d b \hat{c}}-R_{\hat{a} d b \hat{c}}\right\}
\end{gathered}
$$

According the the Definition 2.2, consequently we deduce

$$
\left(G_{R}\right)_{\hat{a} b c \hat{d}}=-\frac{1}{2}\left[A_{b c}^{a d}-3 B^{a d h} B_{h b c}-\frac{5}{3} C^{a d} C_{b c}\right] .
$$

By the same manner, we can get the other components.

Lemma 3.2. In the G-adjoined structure space, the components of the generalized Ricci tensor of NC-manifold are given as the following form:

$$
\left(G_{r}\right)_{\hat{a} b}=\frac{1}{2} A_{c b}^{a c}+3 B^{a c h} B_{h c b}+\frac{5}{3} C^{a c} C_{c b} .
$$

And the others are conjugate to the above component or equal to zero.

Proof: By using the Lemma 2.1 and Definition 2.3, directly we obtain the above components.

Lemma 3.3. In the G-adjoined space, the components of the generalized projective tensor of $\mathrm{NC}$-manifold take the following forms:

(1) $\left(G_{P}\right)_{\hat{a} \hat{b} c d}=-\frac{1}{2 n}\left(\left(\frac{1}{2} A_{f c}^{a f}+3 B^{a f h} B_{h f c}+\frac{5}{3} C^{a f} C_{f c}\right) \delta_{d}^{b}\right)-\left(\frac{1}{2} A_{f c}^{b f}-3 B^{b f h} B_{h f c}-\right.$ $\left.\frac{5}{3} C^{b f} C_{f c}\right) \delta_{d}^{a}$

(2) $\left(G_{P}\right)_{\hat{a} b \hat{c} d}=-A_{b d}^{a c}+\frac{1}{2 n}\left(\frac{1}{2} A_{f b}^{c f}+3 B^{c f h} B_{h f b}+\frac{5}{3} C^{c f} C_{f b}\right) \delta_{d}^{a}$;

(3) $\left(G_{P}\right)_{\hat{a} b c \hat{d}}=-\frac{1}{2 n}\left(\frac{1}{2} A_{b c}^{a d}+3 B^{a d h} B_{h b c}+\frac{5}{3} C^{a d} C_{b c}\right)-\frac{1}{2}\left(A_{f c}^{a f}+3 B^{a f h} B_{h f c}+\right.$ $\left.\frac{5}{3} C^{a f} C_{f c}\right) \delta_{b}^{d}$. 
The remaining components are obtained by taking the conjugated operation to the above components or are identical equal to zero.

Proof:

1. Put $i=\hat{a}, j=\hat{b}, c$ and $l=d$.

According to the Definition 2.4, we obtain

$$
\left(G_{P}\right)_{\hat{a} \hat{b} c d}=\left(G_{\Re}\right)_{\hat{a} \hat{b} c d}-\frac{1}{2 n}\left[\left(G_{r}\right)_{\hat{a} c} g_{\hat{b} d}-\left(G_{r}\right)_{\hat{b} c} g_{\hat{a} d}\right]
$$

By using the Lemmas 3.1, 3.2 and the matrices (2.1), we have

$$
\left(G_{P}\right)_{\hat{a} \hat{b} c d}=
$$

$-\frac{1}{2 n}\left(\left(\frac{1}{2} A_{f c}^{a f}+3 B^{a f h} B_{h f c}+\frac{5}{3} C^{a f} C_{f c}\right) \delta_{d}^{b}\right)-\left(\frac{1}{2} A_{f c}^{b f}-3 B^{b f h} B_{h f c}-\frac{5}{3} C^{b f} C_{f c}\right) \delta_{d}^{a}$.

2. Put $i=\hat{a}, j=b, k=\hat{c}$ and $l=d$.

Harmonize to the Definition 2.4, we get

$$
\left(G_{P}\right)_{\hat{a} b \hat{c} d}=\left(G_{\Re}\right)_{\hat{a} b \hat{c} d}-\frac{1}{2 n}\left[\left(G_{r}\right)_{\hat{a} \hat{c}} g_{b d}-\left(G_{r}\right)_{b \hat{c}} g_{\hat{a} d}\right]
$$

Taking into account the Lemmas 3.1, 3.2 and the matrices (2.1), we obtain

$$
\left(G_{P}\right)_{\hat{a} b \hat{c} d}=-A_{b d}^{a c}+\frac{1}{2 n}\left(\frac{1}{2} A_{f b}^{c f}+3 B^{c f h} B_{h f b}+\frac{5}{3} C^{c f} C_{f b}\right) \delta_{d}^{a} .
$$

By the same technique, we can compute the other components.

Theorem 3.1. If $M$ is vanishing generalized projectively NC-manifold, then the necessary and sufficient condition for $M$ to be vanishing generalized Ricci tensor is the holomorphic tensor vanishes.

Proof: Let $M$ be vanishing generalized projectively $N C$-manifold. According to the Lemma 3.3, we have

$$
\left(G_{\Re}\right)_{\hat{a} b \hat{c} d}+\frac{1}{2 n}\left[\left(G_{r}\right)_{b \hat{c}} g_{\hat{a} d}\right]=0
$$

If $M$ is vanishing generalized Ricci tensor, then directly we get

$$
A_{b d}^{a c}=0 .
$$

Conversely, if $M$ is vanishing holomorphic tensor, then we have

$$
\frac{1}{2 n}\left[\left(G_{r}\right)_{b \hat{c}} \delta_{d}^{a}\right]=0
$$

Contracting 3.2 by the indices $(b, a)$, it follows that

$$
\left(G_{r}\right)_{d \hat{c}}=0 .
$$

Theorem 3.2. An NC-manifold has vanishing holomorphic tensor if and only if, $M$ is a manifold of vanishing generalized projective tensor.

Proof: Let $M$ be $N C$-manifold with vanishing generalized projective tensor. Making use of the Lemma 3.3, we obtain

$$
-A_{b d}^{a c}+\frac{1}{2 n}\left(\frac{1}{2} A_{f b}^{c f}+3 B^{c f h} B_{h f b}+\frac{5}{3} C^{c f} C_{f b}\right) \delta_{d}^{a}=0
$$


Symmetrizing and then antisymmetrizing the equation 3.3 by the indices $(c, f)$, we get

$$
A_{b d}^{a c}=0 .
$$

Conversely, let $M$ be $N C$-manifold with vanishing holomorphic tensor, then the equation (3.3) takes the following formula:

$$
\left(G_{P}\right)_{\hat{a} b \hat{c} d}=\frac{1}{2 n}\left(3 B^{c f h} B_{h f b}+\frac{5}{3} C^{c f} C_{f b}\right) \delta_{d}^{a}
$$

Symmetrizing and then antisymmetrizing the equation (3.4) by the indices $(c, f)$, we deduce

$$
\left(G_{p}\right)_{\hat{a} b \hat{c} d}=0 .
$$

Lemma 3.4. An NC-manifold has $\Phi$-invariant generalized Ricci tensor if and only if,

$$
\Phi \circ G_{r}=G_{r} \circ \Phi .
$$

Theorem 3.3. Let $M$ be $N C$-manifold. Then $M$ has $\Phi$-invariant generalized Ricci tensor if and only if, $\left(G_{r}\right)_{b}^{\hat{a}}=0$ hold in the $G$-adjoined structure space.

Proof: Suppose that $M$ is $\Phi$ - invariant generalized Ricci tensor. According to the Lemma 3.4, we have

$$
\Phi \circ G_{r}=G_{r} \circ \Phi
$$

By the $G$-adjoined structure space, the above equation becomes

$$
\left(\Phi \circ G_{r}\right)_{j}^{i}=\left(G_{r} \circ \Phi\right)_{j}^{i}
$$

This means

$$
\Phi_{k}^{i}\left(G_{r}\right)_{j}^{k}=\left(G_{r}\right)_{k}^{i} \Phi_{j}^{k}
$$

Put $i=\hat{a}$ and $j=b$, then the equation (3.5) becomes

$$
\Phi_{c}^{\hat{a}}\left(G_{r}\right)_{b}^{c}+\Phi_{\hat{c}}^{\hat{a}}\left(G_{r}\right)_{b}^{\hat{c}}+\Phi_{0}^{\hat{a}}\left(G_{r}\right)_{b}^{0}=\left(G_{r}\right)_{c}^{\hat{a}} \Phi_{b}^{c}+\left(G_{r}\right)_{\hat{c}}^{\hat{a}} \Phi_{b}^{\hat{c}}+\left(G_{r}\right)_{0}^{\hat{a}} \Phi_{b}^{0}
$$

By using (2.1), we have

$$
\left(G_{r}\right)_{b}^{\hat{a}}=0 .
$$

Theorem 3.4. Suppose that $M$ is NC-manifold with vanishing generalize projective tensor and $\Phi$-invariant generalized Ricci tensor. Then the necessary and sufficient condition for $M$ to be generalized Einstein manifold is $A_{b d}^{b c}=\frac{G e}{2 n} \delta_{d}^{c}$, where Ge is a generalized Cosmological constant.

Proof: Let $M$ be $N C$-manifold with vanishing generalized projective tensor. According to the Lemma 3.3, we have

$$
-A_{b d}^{a c}+\frac{1}{2 n}\left[\left(G_{r}\right)_{b \hat{c}} g_{\hat{a} d}\right]=0
$$


Making use of the Definition 2.8, the equation (3.6) becomes

$$
A_{b d}^{a c}=\frac{G e}{2 n} \delta_{b}^{c} \delta_{d}^{a}
$$

Contracting the equation (3.7) by the indices $(b, a)$, it follows that

$$
A_{b d}^{b c}=\frac{G e}{2 n} \delta_{d}^{c}
$$

Conversely,

Contracting the equation $(3.6)$ by the indices $(b, a)$, we have

$$
-A_{b d}^{b c}+\frac{1}{2 n}\left[\left(G_{r}\right)_{d \hat{c}}\right]=0
$$

Combining the equations 3.8$)$ and $(3.9)$, we conclude

$$
\left(G_{r}\right)_{d}^{c}=G e \delta_{d}^{c}
$$

Therefore, by the Definition 2.8 and Theorem 3.3, $M$ is generalized Einstein manifold.

Theorem 3.5. Suppose that $M$ is NC-manifold with vanishing generalized Riemannian curvature tensor and $\Phi$-invariant generalized Ricci tensor. If $M$ is a generalized Einstein manifold then $G e=\frac{10}{3} C^{b c} C_{b d}$.

Proof: Let $M$ be $N C$-manifold with vanishing generalized Riemannian curvature tensor. Then from Lemma 3.1 we have

$$
-\frac{1}{2}\left[A_{b c}^{a d}-3 B^{a d h} B_{h b c}-\frac{5}{3} C^{a d} C_{b c}\right]=0
$$

By symmetrization and antisymmetrizatin the equation (3.10) by the induces $(d, h)$ we get

$$
A_{b c}^{a d}-\frac{5}{3} C^{a d} C_{b c}=0
$$

Contracting the equation (3.11)by the induces $(b, a),(d, c)$ and $(c, d)$, we deduce

$$
A_{b d}^{b c}-\frac{5}{3} C^{b c} C_{b d}=0
$$

Since $M$ is generalized Einstein manifold, then from the Theorem 3.4, the equation (3.12) becomes

$$
\frac{G e}{2 n} \delta_{d}^{c}-\frac{5}{3} C^{b c} C_{b d}=0
$$

Contracting the equation (3.13)by the induces $(d, a)$, implies

$$
G e=\frac{10}{3} C^{b c} C_{b d}
$$


Theorem 3.6. 5] Suppose that $M$ is NC-manifold. Then the necessary and sufficient condition that $M$ is a manifold of point constant $\Phi H S$-curvature $C_{0}$ is

$$
A_{b c}^{a d}=B^{a d h} B_{h b c}+\frac{5}{3} C^{a d} C_{b c}+\frac{C_{0}}{2} \tilde{\delta}_{b c}^{a d} .
$$

Theorem 3.7. Suppose that $M$ is NC-manifold of point constant $\Phi H S$-curvature $C_{0}$ and vanishing generalized projective tensor with $\Phi$-invariant generalized Ricci tensor, then $C^{a f} C_{f c}=-\frac{C_{0}(n+1)}{10} \delta_{c}^{a}$.

Proof: Let $M$ be $N C$-manifold of $\Phi H S$-curvature tensor and vanishing generalized projective tensor.

According to the Lemma 3.3, we have

$$
\begin{aligned}
-\frac{1}{2 n}\left(\left(\frac{1}{2} A_{f c}^{a f}+3 B^{a f h} B_{h f c}+\frac{5}{3} C^{a f} C_{f c}\right) \delta_{d}^{b}\right)-\left(\frac{1}{2} A_{f c}^{b f}-\right. & 3 B^{b f h} B_{h f c}- \\
& \left.-\frac{5}{3} C^{b f} C_{f c}\right) \delta_{d}^{a}
\end{aligned}
$$

By using Theorem 3.6, the equation (3.14) becomes

$$
\begin{array}{r}
-\frac{1}{2 n}\left(\frac{1}{2}\left(B^{a f h} B_{h f c}+\frac{5}{3} C^{a f} C_{f c}+\frac{c_{0}}{2} \tilde{\delta}_{f c}^{a f}\right)+3 B^{a f h} B_{h f c}+\frac{5}{3} C^{a f} C_{f c}\right) \delta_{d}^{b}- \\
-\frac{1}{2}\left(-2 B^{b f h} B_{h f c}+\frac{c_{0}}{2} \tilde{\delta}_{f c}^{b f}\right) \delta_{d}^{a}=0
\end{array}
$$

Symmetrizing and then antisymmetrizing the equation 3.15 by the indices $(b, f)$ and $(f, h)$, we conclude that

$$
C^{a f} C_{f c}=-\frac{C_{0}(n+1)}{10} \delta_{c}^{a} .
$$

\section{REFERENCES}

[1] Abood, H. M., Abd Ali, H. G., Geometry of Projective Tensor of Viasman-Gray Manifold, British Journal of Mathematics and Computer Science, V. 14, No. 6, (2016), 1-9.

[2] Abood, H. M., Abd Ali, H. G., Projective-Recurrent Viasman-Gray manifold, Asian Journal of Mathematics and Computer Research, V. 13, No. 3, (2016), 184-191.

[3] Abood, H. M., Holomorphic-geodesic transformation of almost Hermitian manifold, Ph.D. thesis, Moscow state University, Moscow, 2002.

[4] Abood, H. M., Mohammed N. J., Locally Conformal Kähler Manifold of Pointwise Holomorphic Sectional Curvature Tensor, International Mathematical Forum, V. 5, No. 45, (2010), $2213-2224$

[5] Abood, H. M., Mohammed N. J., Nearly Cosymplectic Manifold of Holomorphic Sectional Curvature Tensor, Far East Journal of Mathematical Science, V. 106, No. 1, (2018), 171-181.

[6] Atceken, M., On Generalized Sasakian Space Forms Satisfying Certain Conditions On The Concircular Curvature Tensor, Bulletain of Mathematical Analysis and Applications, V. 6, (2014), 1-8.

[7] Atceken, M., Yildirim U., Almost $C(\alpha)$-manifolds Satisfying Certain Conditions, Advanced Studies in Contemporary Mathematics, V. 26, N. 3, (2016), 567-578.

[8] Blair, D. E., The Theory of Quasi - Sasakian Structures, J. Differential Geometry, No. 1, (1967), 331-345. 
[9] Blair, D.E., Showders D.K., Yano K., Nearly Sasakian Structure, Kodoi Math. Sem. Rep. 27, No. 1-2, (1976), 175-180.

[10] Gan, G., Characteristic of Some Classes of Almost Hermitian Manifolds, Serdica, V. 4, (1978), $19-23$.

[11] Kirichenko, V. F., Differential geometry of K-space, Problems of Geometry, V.8, (1977), 139-160.

[12] Kirichenko, V. F., Sur le geometrie des varietes approximativent cosymlectiques, C. R. Acad. Sci. Paris, 295, (1983), 673-676.

[13] Kirichenko, V. F., The Method of Generalization of Hermitian Geometry in The Almost Hermitian Contact Manifold, Problems of geometry VINITE ANSSR , V. 18, (1986), 25-71.

[14] Kirichenko, V. F., Differential - Geometry Structures on Manifolds, Second edition, expanded. Odessa, Printing House, p. 458, 2013.

[15] Kirichenko, V. F. and Kusova, E. V., On Geometry of Weakly Co-symplectic Manifold, Journal of Mathematical Sciences, 177, (2011), 668.

[16] Kirichenko, V. F. and Rustanov, A. R. Differential Geometry of Quasi-Sasakian Manifolds, Mathematical Collection, V. 193, No. 8, (2002), 71-100.

[17] Kobayashi, S. and Nomizu, K., Foundations of Differential Geometry, John Wily and Sons, V.1, 1963.

[18] Mileva, P., Locally Conformally Kähler Manifolds of Constant Type and J-Invariant Curvature Tensor, Facta universitatis, Series: Mechanics, Automatic control and Robotics, V. 3, No. 14, (2003), 791-804.

[19] Petrov, A. Z., Einstein Space, Phys-Math. Letr., Moscow, (1961), p. 463.

[20] Shashikala, S., Venkatesha, On Generalized Psedo-Projective $\Phi$-Recurrent $(k)$-Contact Metric Manifold, International $J$. of scientific and research puplications, V.4, Issue 3, (2014), 22503153.

[21] Yildirim, U., Atceken, M., On The $C(\alpha)$-manifolds Satisfying Certain Conditions On QuasiConformal Curvature Tensor, Proceding of the Jangjeon Mathematical Society, V.19, N. 1, (2016), p.115-124.

[22] Yildirim, U., Atceken, M. and Dirik, S., Pseudo Projective Curvature Tensor Satisfying Some Properties On A Normal Paracontact Metric Manifold, Commun. Fac. Sci. Univ. Ank. Ser. A1 Math. Stat., V. 68, No.1, (2019), 997-1016.

Current address: Nawaf J. Mohammed, University of Basrah, Department of Mathematics, Basra, IRAQ

E-mail address: nawafjaber80@yahoo.com

ORCID Address: http://orcid.org/0000-0002-5426-1447

Current address: Habeeb M. Abood: University of Basrah, Department of Mathematics, Basra, IRAQ

E-mail address: iraqsafwan2006@gmail.com

ORCID Address: http://orcid.org/0000-0002-3257-9550 